\title{
Formal Method of Encryption Using 9'S Complement
}

\author{
Srinivasan Nagaraj \\ Asst. Professor \\ Dept. of CSE, GMRIT \\ Rajam - 532127, AP, India
}

\author{
Kishore Bhamidipati \\ Asst. Professor \\ Dept. of IT, GMRIT \\ Rajam - 532127, AP, India
}

\author{
M Ramachandra \\ Asst. Professor \\ Dept. of CSE, GMRIT \\ Rajam - 532127, AP, India
}

\begin{abstract}
In this paper we show how implemented one time security encryption scheme is more lucid, effective. However it is more complex in nature of attacker view. The one-time pad encryption scheme itself is mathematically unbreakable. (See Claude Shannon's "Communication Theory of Secrecy Systems"). Therefore, the attacker will focus on breaking the key instead of the cipher text. Random key stream can be used to create lifetime supply of keys for one time pads. Here we provided the practical approach that you can use to set up your own one-time pad encryption. Permutation techniques can be used in conjunction with other technique includes substitution, encryption function etc. for effective performance. The goal of this paper to show how the one-time pad encryption technique can be achieved including complement approach technique.
\end{abstract}

\section{Keywords}

Cryptography, One-time pad, Encryption, Key-Enhancement, Network Security, Computer Security.

\section{INTRODUCTION}

Computer tend now a days to be organized in large net-works connected to Internet, and are used in an increasing number of sensitive applications, such as online banking and shopping, control of hardware installations and public infra-structure. Meanwhile, as more and more critical tasks are delegated to computers, computer security becomes a matter of first importance, hence the interest of understanding what everyday practical computer security consist.

The Network security is concerned with the security of information. [Good security means that the system and users are protected from attacks originating from inside the network just as well as they from outside attacks.] Security - guarding against interference by entities external to a system. The main aim is to protect the information, which is sent from one computer to another computer through network. The information security is defined as follows.

Information security $=$ Confidentiality + integrity + availability + authentication. There can be no information security without confidentiality. Confidentiality ensures the unauthorized users do not intercept, copy or replicate the information. The integrity is necessary so that the accurate information can flow over the network. The information security is also required during the retrial of the data. The users should be authenticated to retrieve data and the information is not secure without authentication.

There is no such thing as a completely secure computer network. The nature of a network is to allow communication.
Any communication can fall into the wrong hands. The purpose of this guide is to help you secure your network without putting a halt to its use and put in place the safeguards to detect when your security is breached.

Today's corporate networks are complex and diverse. They connect mainframes, minis, PC's, LAN's and peripherals over ever-widening geographic boundaries. This diversity, both technically and geographically, means that devising an effective corporate-wide security plan involves adapting security techniques and procedures from the various systems currently incorporated in your company. Control access to your network and all its related parts. (This means terminals, switches, gateways, bridges, routers, even printers).

\subsection{Objectives of Network Security}

Ensure that any message sent, arrives at the proper destination. Ensure that any message received was in fact the one that was sent (nothing added or deleted) they refer to as Minimal Security Functional Requirements for Multi-User Operational Systems. The major functions are listed below.

Protect information in-transit, from being seen, altered, or removed by an unauthorized person or device.

Network security is the effort to create a secure computing platform, designed so that agents (users or programs) cannot perform actions that they are not allowed to perform, but can perform the actions that they are allowed to. The actions in question can be reduced to operations of access, modification and deletion. Network security can be seen as a subfield of security engineering, which looks at broader security issues in addition to network security.

\subsection{One Time Pad}

In cryptography, the one-time pad (OTP) is a type of encryption, which has been proven to be impossible to crack if used correctly. Each bit or character from the plaintext is encrypted by a modular addition with a bit or character from a secret random key (or pad) of the same length as the plaintext, resulting in a ciphertext. If the key is truly random, as large as or greater than the plaintext, never reused in whole or part, and kept secret, the ciphertext will be impossible to decrypt or break without knowing the key.

One well known realization of perfect secrecy is the One-time Pad, which was first decscibed by Gillbert Vernam in 1917 for use in automatic encryption and decryption of Tele-graph messages. It is interesting that the One-time Pad was though for many years to be an "unbreakable" cryptosystem, but there was no mathematical proof of this until Shannon developed the concept of perfect secrecy over 30 year later. We can only talk about OTP if four important rules are followed. If these rules are applied correctly, the one-time pad can be proven to be unbreakable (see Claude Shannon's "Communication Theory of 
Secrecy Systems"). However, if only one of these rules is disregarded, the cipher is no longer unbreakable.

1. The key is as long as the plaintext.

2. The key is truly random (not generated by simple computer Rnd functions or whatever!)

3. There should only be two copies of the key: one for the sender and one for the receiver (some exceptions exist for multiple receivers)

4. The keys are used only once, and both sender and receiver must destroy their key after use.

For practical application, the key used for one time pad cipher is a string of random bits, usually generated by a Cryptographically Strong Pseudo-Random Number Generator. However for ultimate security, it is suggested to generate the key by using the natural randomness of quantum mechanical events, if the key is truly random an XOR operation based one -time pad encryption scheme is perfectly secure against cipher text-only cryptanalysis. We come to the point that if the hackers do not know the sender or receiver key, then the one time pad encryption scheme is $100 \%$ secure. A one-time pad is essentially a pad of paper on which each page has a unique set of random letters. The sender and receiver have identical pads. Each letter on the pad is used to determine a single letter of the enciphered message. Since the letters on the pad are random, there is no formula that can be determined by studying the letters. Assuming that the pad is not compromised, and each page is used only once, then the OTP system is unbreakable. One-time pads are "informationtheoretically secure" in that the encrypted message (i.e., the cipher text) provides no information about the original message to a cryptanalyst (except the length of the message). This is a very strong notion of security first developed during WWII by Claude Shannon and proved, mathematically, to be true of the one-time pad by Shannon about the same time. His result was published in the Bell Labs Technical Journal in 1949. Properly used one-time pads are secure in this sense even against adversaries with infinite computational power.

\section{MEDTHODOLOGY}

In this article we consider the basic fact of one time pad encryption scheme with little bit concept of 9's complement is added and then evaluate the scheme. Here we use concept like key size must be same as long as the plaintext. Key must be truly random and at last keys are used only once, and both sender and receiver must destroy their key after use. Here we first create the chart for alphabet 1-26 that shown in Table-1 and then take the decimal equivalent value of the text message from table then take the 9's complement of decimal value convert the 9's complemented decimal value in to 6 bit binary, generate the random number key, then after apply XOR properties, [i.e. $1-1=0,0-0=0$ and $1-0=1,0-1=1$ ] after xoring take the 1' complement of result, after that convert the complement value into decimal digit, if digit value is more that 26 then subtract the value form 26 and write equivalent alphabet which is the encrypted message sent to the recipient, then recipient apply reverse process and get the plain text.

\section{Table 1}

\section{Alphabet Number Binary 6 bit Eq. Decimal Value}

000001
000010

3

000011

000100

4

000101

000110

6

000111

7

001000

001001

001010

10

001011

11

001100

001101

13

001110

001111

15

010000

16

010001

17

010010

18

010011

19

010100

20

010101

010110

010111

011000

24

011001

25

011010

\section{ALGORITHM}

Steps as follows:

1. Consider the Plain text

2. Take decimal value of the text message from table

3. Take 9's complement of the decimal value

4. Convert 9's complemented value into 6 bit binary.

5. Apply 6 bit key

6. Apply property of XOR

7. Take the 1's complement of resulted XOR

8. Convert XOR resulted value into decimal. If decimal value is more than 26 subtract it from 26 and then write equivalent alphabet from table shown.

9. For step 8, generate a string with values such that, if decimal is subtracted at the corresponding location put ' 1 ' else put ' 0 '.

10. Attach this string to the alphabetical text.

11. Send this encrypted to the other side or to the receiver.

12. Split the alphabetical text and numbers.

13. For alphabetical text write the decimal values.

14. Now apply the operations as below.

$$
\mathrm{D}(\mathrm{i})=\mathrm{E}(\mathrm{i})+\mathrm{N}(\mathrm{i}) * 26
$$

15. Now convert $\mathrm{D}$ into 6 bit binary from table.

16. Apply the same key and XOR Properties. 
17. Take the 1's and 9's complement

18. Convert into equivalent alphabet which is not but the plain text.

\section{IMPLEMENTATION AND ANALYSIS}

Here we consider one simple example for wide understanding Encrypted Process from Sender Side:-

Take the example here as a plain text GOD

G $\quad 0 \quad$ D

Decimal equivalent from table $\mathrm{G}=7, \mathrm{O}=15$ and $\mathrm{D}=4$ Take the 9's complement i.e. 9-7=2, 9-1 =8, 9-5 =4 and 9-4 =5 So $\begin{array}{lllll} & 2 & 8 & 4 & 5\end{array}$

Convert 6 bit binary and apply 6 bit key

$\begin{array}{lcccc}6 \text { bit } & 000010 & 001000 & 000100 & 000101 \\ \text { Key } & 101010 & 101010 & 101010 & 101010 \\ \text { X-OR } & 101000 & 100010 & 101110 & 101111 \\ \text { 1's Compl: } & 010111 & 011101 & 010001 & 010000 \\ \text { Decimal } & 23 & 3 & 17 & 16\end{array}$

Equi Alphabet from table w c q p

\section{Encrypted text is WCQP of GOD}

This encrypted text WCQP and corresponding values are 0100 is send to the recipient

Decrypted Process from Recipient Side

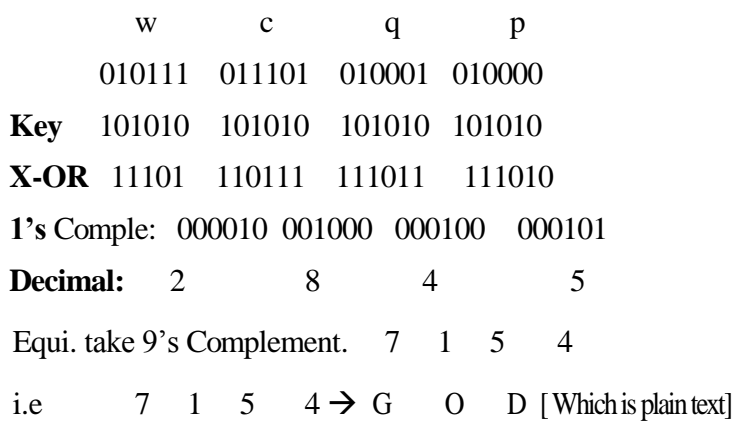

\section{CONCLUSION}

In this paper showed that how we implemented one time security and This scheme is more lucid for user point of view but very difficult for attacker point of view. Here we are using 9's and 1's complement for making more complexity for attacker side, so attacker could not find as well as not guessing the plain text easily. So this scheme is more applicable for sensitive application, Again the key is used is same as well as different for all text so it is beneficial for user from both side.

\section{REFERENCES}

[1] Douglas R, Stinson “ CRYPTOGRPHY Theory and Practice " Second Edition.

[2] Charlie Kaufman st al. "Network Security "PRIVATE Communication in a PUBLIC World., Prentice Hall of India Private Limited. 2003.

[3] Information Technology Journal 4(3) : 204-221,2005

[4] Claude Shannon's "Communication Theory of Secrecy Systems".

[5] Thomas L. Floyd "Digital Fundamentals".

[6] Neal R. Wagner "The Laws of Cryptography: Perfect Cryptography: The One-Time Pad".

[7] Ritter, Terry 1991. The Efficient Generation of Cryptographic Confusion Sequences. Cryptologia "15: 81$139 "$.

[8] Pete McCollum "Encryption Via One-Time Pads".

[9] Erskine, Ralph, "Enigma's Security: What the Germans Really Knew", in "Action this Day", edited by Ralph Erskine and Michael Smith,. 